\section{Ignazio Gallo}

ignazio.gallo@uninsubria.it

Alessandro Zamberletti

a.zamberletti@uninsubria.it

Simone Albertini

simone.albertini@uninsubria.it

Lucia Noce

lucia.noce@uninsubria.it
Applied Recognition Technology Laboratory Department of Theoretical and Applied Science University of Insubria Varese, Italy

\section{Overview and Results}

In this paper we approach the task of figure-ground segmentation of natural images using a novel framework to generate highly collaborative tree-based structures, called High Entropy Ensembles (HEE). While many model combination frameworks adopt rejection rules to improve the classification time of the ensembles at the cost of restricting the interactions between the different elements in the structures, throughout our work we prove that, similarly to the Cascade Classification Model [3], when execution time is not critical, better results can be obtained when encouraging that kind of interaction by combining heterogeneous suboptimal classifiers into highly connected tree-based ensembles in which the different algorithms communicate with each other to let the strengths of one overcome the weaknesses of the others and vice versa. Inspired by randombased model combination approaches [2], we do not focus on looking for the optimal classifiers to be added to the HEE, instead we pick them from a pool of randomly configured segmentation algorithms. This randomness injection increases the effectiveness of HEE while also decreasing both the computational complexity of the model creation procedure and the risk of overfitting the training data, which is a common issue for most model combination frameworks.

\section{Proposed Method}

The proposed method consists in a building phase that creates a figureground segmentation ensemble by executing an initial base step followed by a recursive sequence of bottom-up and top-down steps. The building procedure is driven by the maximization of a goodness function that defines the quality of the HEE being built. The goal of the initial base step is to identify both the first suboptimal figure-ground segmentation algorithm $a$ that needs to be added to the ensemble $T$ and its set of input image features $F_{a}$, as shown in Fig. 1a. Once the first node has been identified and added to the structure, the ensemble $T$ is progressively augmented by adding new suboptimal root and leaf nodes through a recursive sequence of bottom-up and top-down steps, as shown in Fig. 1b and Fig. 1c respectively. The building phase terminates once a bottom-up step followed by a top-down step do not increase the value of the goodness function computed for $T$. The resulting HEE $T$ can be used to generate the soft figure-ground segmentation map $M_{I}^{t_{r}}$ for a given image $I$ simply by providing $I$ as input to every node in $T$, as summarized in Fig. 1d. The final binary segmentation map $M_{I}$ for $I$ is obtained by thresholding $M_{I}^{t_{r}}$.

\section{Results}

An end-to-end experimental analysis is conducted in order to compare HEE against other state-of-the-art figure-ground segmentation algorithms and model combination methods on several challenging datasets: Weizmann Horses, Oxford Flowers 17, INRIA Graz-02 and the figure-ground variant of Pascal VOC 2010; despite the simplicity of our approach, HEE outperform all the evaluated competing methods.

\section{Conclusion}

The proposed method does not require any user input nor extensive tuning and constitutes a valid alternative to other frameworks when combining heterogeneous figure-ground segmentation algorithms. It is particularly interesting to observe that in many cases the set of image features automatically selected by the building phase as input features for the nodes in

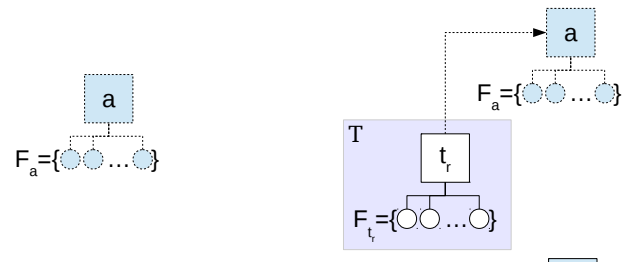

(a) (c)
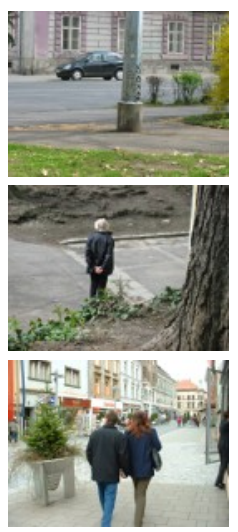

I

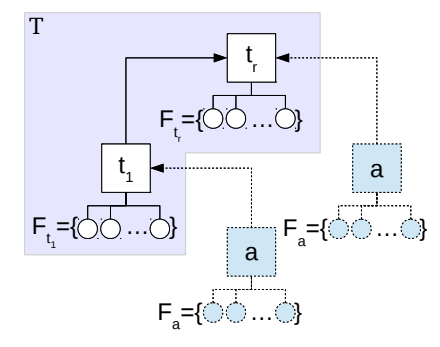

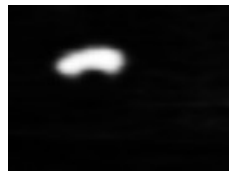
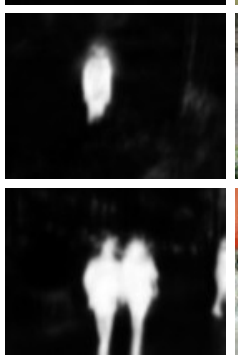

$M_{1}^{t}$

(e)
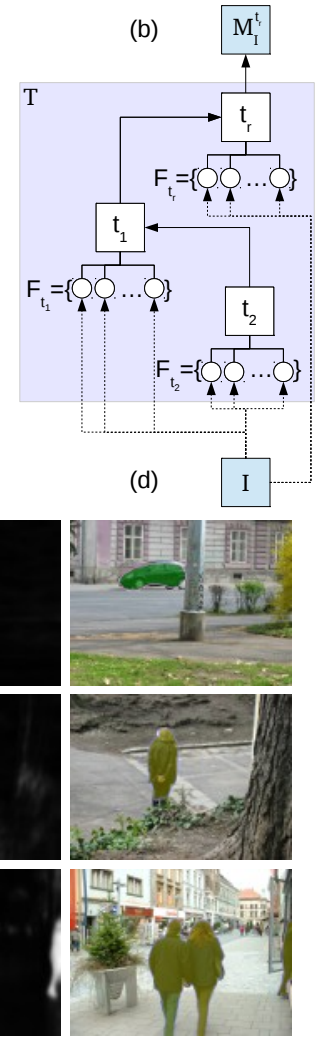

$M_{1}$
Figure 1: HEE building procedure and segmentation examples. (a) base step (b) bottom-up step (c) top-down step (d) segmentation of an image $I$ (e) segmentation examples for images from INRIA Graz-02.

the HEE resembles the base set of Integral Channel Features [1] (LUV, gradient histogram and magnitude) widely used by state-of-the-art rigid object detection algorithms. This proves that, even tough the proposed model is heavily random-based, it tries to build optimal segmentation ensembles. It is an open question whether our method can pose a challenge to other similar approaches when applied to more challenging tasks, such as object classification or multi-class image segmentation.

[1] P. Dollár, Z. Tu, P. Perona, and S. Belongie. Integral channel features. In British Machine Vision Conference, 2009.

[2] Pierre Geurts, Damien Ernst, and Louis Wehenkel. Extremely randomized trees. Machine Learning, 63(1):3-42, 2006.

[3] Geremy Heitz, Stephen Gould, Ashutosh Saxena, and Daphne Koller. Cascaded classification models: Combining models for holistic scene understanding. In International Conference on Neural Information Processing Systems, 2008. 